\title{
Estudio experimental del efecto de la excitación basal y grado de saturación en las presiones de poros bajo una presa de tierra
}

\author{
Experimental study of the basal excitation and saturation degree effect on the pore pressure \\ underneath an earth dam \\ Fecha de entrega: 20 de abril 2017 \\ Fecha de aceptación: 24 de agosto 2018
}

\section{Mario Pastén ${ }^{1}$ e Iván Salazar ${ }^{2}$ \\ ${ }^{1}$ FCA Ingeniería, Borgoño 934, Oficina 503, Edificio las Empresas, Antofagasta, Chile, mpasten@fcaingenieria.cl \\ ${ }^{2}$ Departamento de Ingeniería Civil, Universidad Católica del Norte, Avda. Angamos 0610, Antofagasta, Chile, isalazar@ucn.cl}

Se investiga la variación de las presiones de poros y el grado de saturación en régimen transiente bajo una presa de tierra cuando dicha estructura es sometida a excitaciones dinámicas basales. Se construye un modelo fisico en el laboratorio de una presa de tierra a escala reducida (1:200). Se utilizan rangos de frecuencias de 3 a $10 \mathrm{~Hz}$, dirección vertical y horizontal, y funciones seno, coseno $y$ triangulares para representar la excitación dinámica basal de la estructura. El control del proceso experimental es llevado a cabo con el registro continuo de las presiones de poros, junto con un balance hídrico general que permite controlar tanto la capacidad de infiltración del sistema como el almacenamiento interno de agua y caudal de salida. Se obtienen resultados de presión de poros que reflejan la magnitud de la oscilación piezométrica. Se encontró que las presiones de poros presentan mayores valores para un alto grado de saturación del medio y para bajas frecuencias de excitación. Además, se asocian cambios permanentes de la presión de poros debido a sobrecargas y succiones producidas en la interfaz presa-suelo de fundación, como consecuencia de las excitaciones basales impuestas.

Palabras clave: presa de tierra, presiones de poro, grado de saturación, frecuencia de excitación, flujo subterráneo
The pore pressure variation and the degree of saturation in a transient regime under an earth dam structure containing liquid are investigated, when subjected to basal dynamic excitations. A scaled physical model (1:200) of an earth dam is built. Frequency ranges between 3 and $10 \mathrm{~Hz}$, in the vertical and the horizontal direction are used. Sine, cosine and triangular functions are used to represent the basal excitation of the structure. The experimental control process is carried out with the continuous measurement of the pore pressure along with a general hydrological balance, which allows controlling not only the infiltration capacity of the system but also the internal storage of water and effluent flow. Results of pore pressure represent piezometric level magnitudes. It was found that larger pore pressure values occur with the increase of the medium degree of saturation and low excitation frequencies. Also permanent changes in pore pressure due to surcharges and suction produced in the dam-foundation soil interface are observed, as a consequence of the imposed ground excitations.

Keywords: earth dam, pore pressure, degree of saturation, excitation frequency, seepage

\section{Introducción}

El estudio del proceso de infiltración en las obras de contención de líquidos como presas de tierra y presas de relaves ha cobrado importancia en los últimos años debido a que tales obras son de gran magnitud y pueden verse sometidas a fuertes cargas cíclicas. Por lo general, en el caso de presas de relaves, los estudios experimentales se realizan a través de ensayos puntuales, metodologías bajo condiciones ideales y acotado a un número de ensayos (e.g. Valenzuela, 2016; Valenzuela et al., 2016).
Si bien es cierto la literatura asociada a la infiltración en medios porosos es bien extensa y profunda en cuanto a metodologías y análisis matemático, existe una escasez de información experimental y más aún al incorporar una componente dinámica al proceso de infiltración, la cual matemáticamente es difícil de relacionar. No obstante, existen estudios asociados a otros escenarios similares como la relación entre eventos dinámicos en zonas de falla tectónica y flujos subterráneos, especialmente hidrotermales. Sibson et al. (1975) estudia un proceso de la 
interacción entre tensiones tanto de rotura como tectónicas del medio y las presiones de poros, al cual denomina como bombeo sísmico. Dicho proceso consta de 3 fases como efectos de la concentración de tensiones tectónicas (Figura 1):

1) Inicio de la dilatación mediante grietas, originando una caída de presión $P$ a causa de la nueva porosidad producida, generando un aumento de la resistencia a la rotura del suelo $\tau_{\mathrm{f}}$

2) Relleno de grietas con el fluido de tal forma que las presiones de poros aumentan y a su vez disminuye la tensión de rotura.

3) Caída de la tensión tectónica $t$ a través del evento sísmico, que genera un relajo de las grietas y el fluido migra a la dirección aliviadora de la presión.

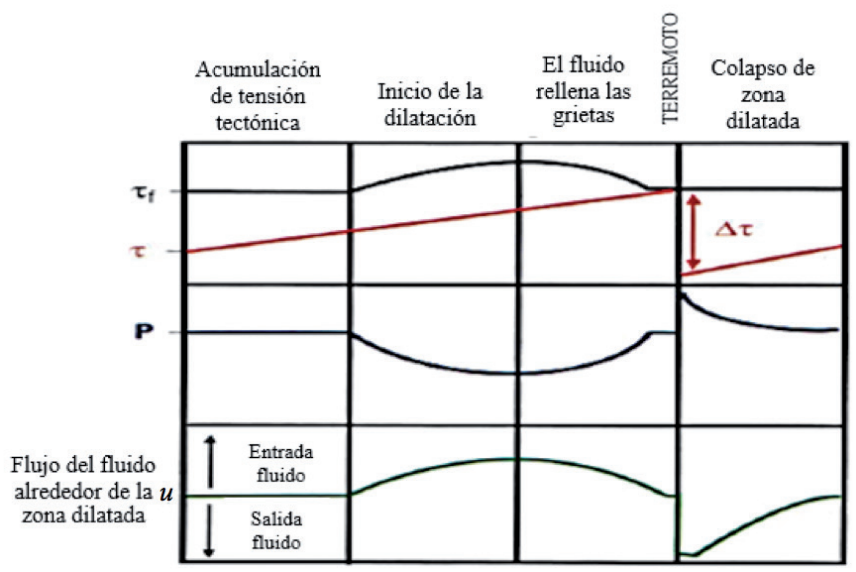

Figura 1: Esquema de interacción entre tensiones tectónicas $\tau$ y de rotura del suelo $\tau_{\mathrm{f}} \mathrm{y}$ presiones de poros $u$ de los fluidos contenidos (Martínez, 2010)

Siguiendo los antecedentes de flujos hidrotermales, Shibata y Akita (2001) enfocan un estudio a erupciones volcánicas, específicamente a la variación de las presiones de poros antes, durante y después de la erupción del volcán Usu en Japón, aunque a nivel regional. Este estudio constata el decaimiento de las presiones de poros previas al momento de la erupción. Se destaca el hecho que a medida que se acerca al día de la erupción, la tasa de decaimiento va en aumento hasta caer bruscamente 2 mca (metros de columna de agua), el día del evento. Por otro lado, otros autores asocian la influencia de determinadas magnitudes sísmicas en la generación de oscilaciones piezométricas. Roeloffs (1998) establece que existe una mayor sensibilidad en las variaciones piezométricas cuando las magnitudes sísmicas son iguales o mayores a $0.69+2.45 \log D$, en la cual $D$ es la distancia desde un sondaje al hipocentro en $\mathrm{km}$. Por otra parte, Rojctazer y Wolf (1989) apuntan a un aumento de permeabilidad del medio y cambio en los caudales de la fuente, tras el sismo de Loma Prieta, EE.UU. Koizumi et al. (1996) presentan un caso similar al de los autores anteriores, con variación de presiones post sísmicas y cambios de flujos en Hyogo-Ken Nanbu, Japón.

Las oscilaciones piezométricas se manifiestan de varias formas. Kissin et al. (1996) presentan casos de variación de presiones como las mostradas en la Figura 2. Por ejemplo, las variaciones con retorno al nivel inicial (Figura 2-A), otras con descensos bruscos y sin retorno (Figura 2-B), variaciones progresivas (Figuras 2-C, D, E) y oscilaciones instantáneas (Figura 2-F). Kissin et al. (1996) establecen que estas variaciones son respuestas frente a tensiones extensivas y compresivas o movimientos a lo largo de la falla y también consideran que el retorno de algunas oscilaciones al nivel inicial es asociado a la elasticidad del terreno.

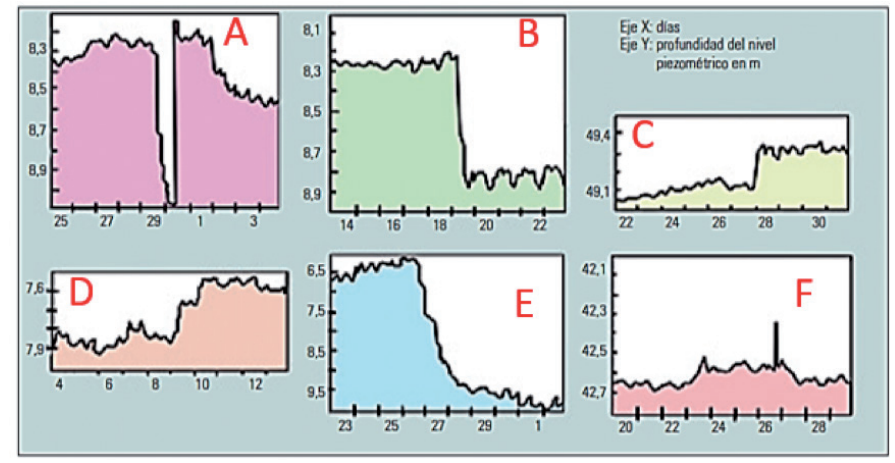

Figura 2: Tipos de variaciones de presión ante fallas activas (Martínez, 2010)

Este trabajo surge producto de la alta sismicidad que existe en Chile, donde las presas de tierra y de relaves se han visto enfrentadas a eventos sísmicos significativos (e.g. Campaña et al., 2010; Figueroa et al., 2017). Por lo tanto, es necesario estudiar los posibles efectos de la actividad sísmica en los fenómenos de escurrimiento que afecten la estabilidad de la presa. Este artículo presenta el diseño y construcción de un modelo experimental a escala de una presa y resultados preliminares de presiones de poros bajo la presa como consecuencia de la imposición de diversos tipos de excitaciones basales. 


\section{Diseño y construcción del modelo experimental}

El modelo experimental mostrado en la Figura 3, se basa en un sistema de flujo bidireccional que simula la infiltración en una presa de relaves. Dicho modelo consta de un muro de taludes 2.5:1 (H:V), construido con la fracción gruesa del material de relave. El modelo a escala posee un núcleo de hormigón que otorga rigidez a la presa. Cabe hacer notar que la construcción de núcleos impermeables se realiza por lo general en presas de tierra para impermeabilizar y así reducir el flujo de agua a través de la presa. Sin embargo, en presas de relaves no es común el uso de núcleos impermeabilizantes o rigidizantes. En la zona del agua embalsada fue colocada una capa de lamas (fracción fina del relave) en forma espesada de acuerdo al proceso constructivo (e.g. Valenzuela, 2016). La estructura se apoya sobre un suelo base de $15 \mathrm{~cm}$ de espesor, correspondiente a una arena mal gradada con poca cantidad de finos. La altura de agua detrás de la presa se mantiene constante durante el ensayo.

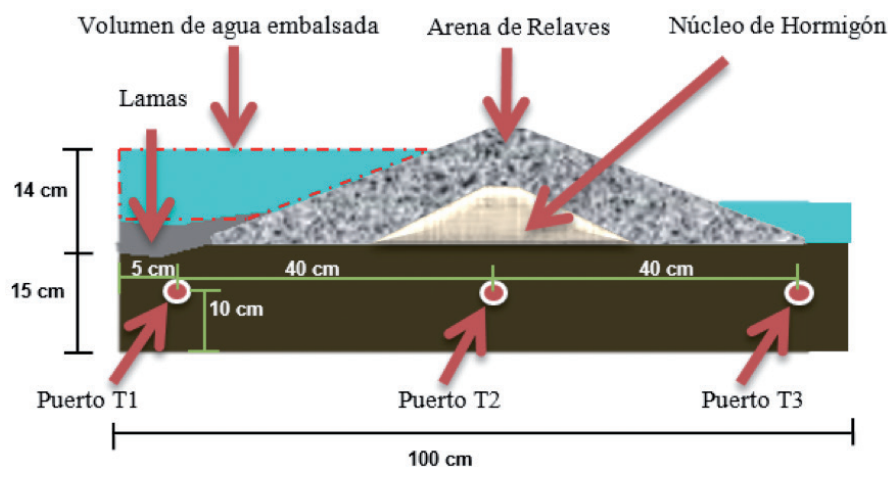

Figura 3: Esquema de elementos y dimensiones del modelo experimental (Pastén, 2016)

Para determinar el gradiente hidráulico y detectar la transición entre la zona saturada y no saturada, se colocaron transductores de presión de poros a lo largo de la presa, en dirección del flujo subterráneo, tal como se indica en la Figura 3 como Puerto 1, 2 y 3 y que también se muestra en la Figura 4a.

Para la generación del movimiento dinámico basal fue utilizada la plataforma vibradora MOOG 62F2000E mostrada en la Figura 4b. Este equipo está constituido por una plataforma rígida de acero y cubierta de madera. Cuenta con pistones hidráulicos que permiten activar 6 grados de libertad de movimientos, desplazamientos en los 3 ejes ortogonales y los respectivos giros a los ejes mencionados. El sistema es operado a través de una interfaz externa, en el cual se elige o se crea la función deseada y se envía al computador interno de la plataforma a través del software SmartFTP. Para mayores antecedentes sobre este equipo consultar Pastén (2016).
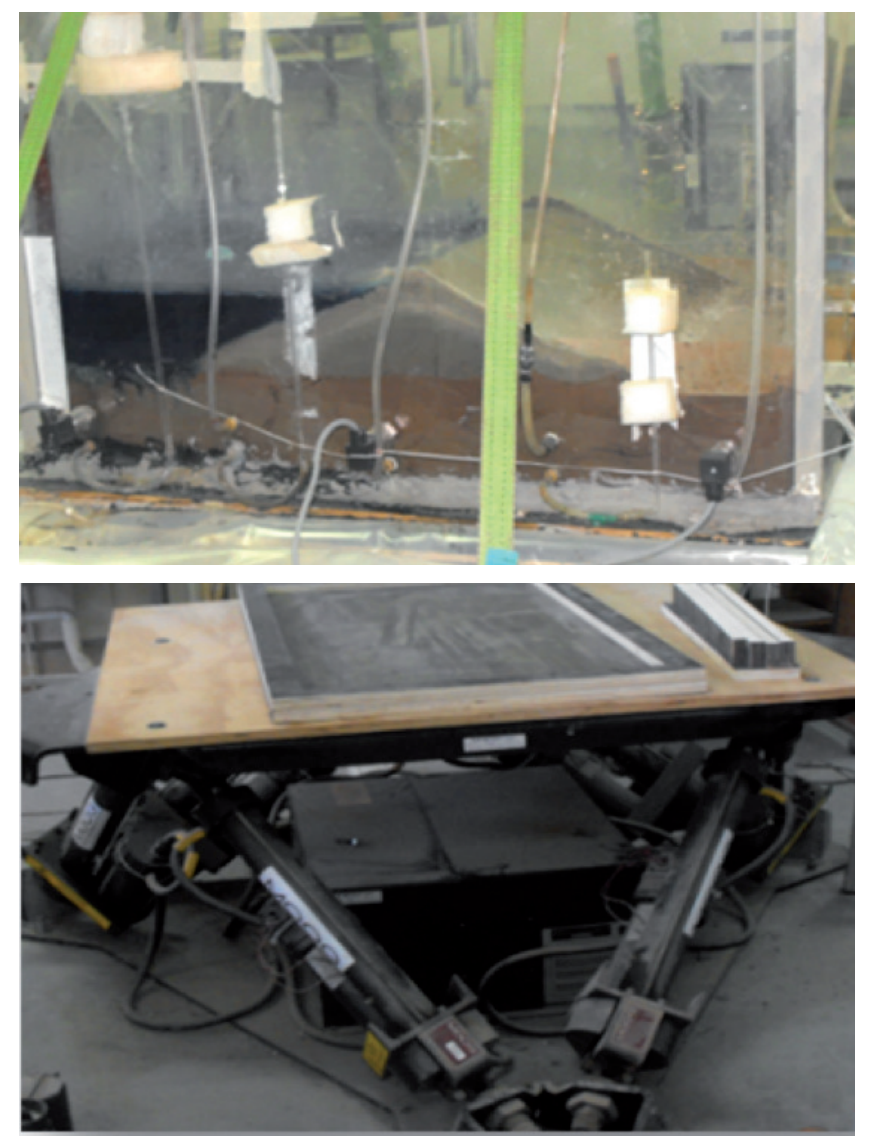

Figura 4: a) Modelo físico mostrando transductores de presión localizados al costado del tranque y b) plataforma MOOG 62F2000E

\section{Medición de volúmenes de agua y presiones de poros}

En la Figura 5 se presenta un diagrama de flujo de la rutina experimental para las mediciones. El proceso de infiltración queda controlado a través de un balance hídrico generado por la interacción entre el volumen infiltrado y el volumen de agua drenada aguas debajo de la presa. Las presiones de poros registradas en los transductores son recopiladas a través de dataloggers con entrada USB, los cuales se configuran mediante el software Easylog USB versión 5.53 para establecer los intervalos de tiempo de registro, límites de presiones máximas y mínimas y los tiempos de entrada y salida. 


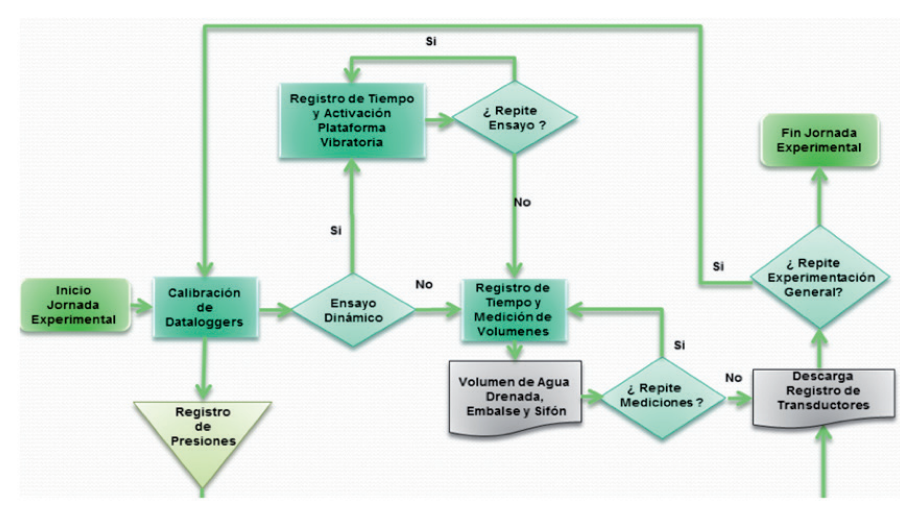

Figura 5: Diagramas de flujo de la rutina experimental

\section{Operación de plataforma vibradora}

Las funciones de excitación se pueden crear por medio de una rutina implementada en el equipo, por ejemplo, funciones sinusoidales y triangulares. Estas funciones sólo necesitan 2 parámetros para ser creadas en la rutina, estas son la frecuencia y amplitud de desplazamiento. La relación de los parámetros enunciados anteriormente y la aceleración del modelo queda estipulada bajo la siguiente expresión:

$$
a=\Delta_{\text {desp }} \omega^{2}
$$

donde $a\left[\mathrm{LT}^{-2}\right]$ es la aceleración producto de la excitación en la base, $\Delta_{\text {desp }}[\mathrm{L}]$ es el desplazamiento de la base y $\omega\left[\mathrm{T}^{-}\right.$ 1] la frecuencia de la excitación. Se utilizaron las funciones clásicas seno, coseno y triangulares incorporadas en la mesa vibradora. Además, se utilizaron rangos de frecuencias de 3 a $10 \mathrm{~Hz}$ tanto en la dirección vertical como en la horizontal. Lo anterior se escogió para que la mesa vibradora se moviera en forma continua y no discreta y así simulara un movimiento dinámico continuo en la base. Las funciones utilizadas se indican en la Tabla 1 y la Figura 6 muestra la ventana de ingreso de funciones de excitación consideradas en una rutina.

\section{Resultado de ensayo estático}

Para la calibración del modelo experimental se muestran en la Figura 7 los resultados obtenidos de las mediciones para un periodo de 3 días $\left(2.6 \cdot 10^{5} \mathrm{~s}\right)$. La Figura 7 a muestra la variación de las presiones de poros medidas en los tres puntos bajo la presa para los 3 días y la Figura $7 \mathrm{~b}$ muestra los resultados en las primeras 2 horas.
Tabla 1: Funciones de excitación en el modelo (perturbaciones)

\begin{tabular}{|c|c|c|c|c|}
\hline $\begin{array}{c}\text { Nombre } \\
\text { función }\end{array}$ & $\begin{array}{c}\text { Dirección } \\
\text { desplazamiento }\end{array}$ & $\begin{array}{c}\text { Fun- } \\
\text { ción }\end{array}$ & $\begin{array}{c}\text { Amplitud } \\
\text { desplazamiento } \\
\Delta_{\text {desp }}, \mathrm{mm} / \mathrm{pulg}\end{array}$ & $\begin{array}{c}\text { Frecuencia } \\
\omega, \mathrm{Hz}\end{array}$ \\
\hline $\mathrm{f} 606$ & $\rightarrow$ & $\mathrm{sen}$ & $1.6 / 0.061$ & 8 \\
\hline $\mathrm{f} 607$ & $\rightarrow$ & $\mathrm{sen}$ & $1.0 / 0.039$ & 10 \\
\hline $\mathrm{f} 608$ & $\uparrow$ & $\mathrm{sen}$ & $1.6 / 0.061$ & 8 \\
\hline $\mathrm{f} 609$ & $\uparrow$ & $\mathrm{sen}$ & $1.0 / 0.039$ & 10 \\
\hline $\mathrm{f} 610$ & $\rightarrow$ & $\mathrm{sen}$ & $2.0 / 0.080$ & 7 \\
\hline $\mathrm{f} 612$ & $\uparrow$ & sen & $2.0 / 0.080$ & 7 \\
\hline $\mathrm{f} 615$ & $\mathrm{x} \rightarrow \mathrm{z} \uparrow$ & $\begin{array}{c}\text { sen/ } \\
\text { cos }\end{array}$ & $2.0 / 0.080$ & 7 \\
\hline $\mathrm{f} 620$ & $\rightarrow$ & sen & $11.0 / 0.435$ & 3 \\
\hline $\mathrm{f} 621$ & $\uparrow$ & sen & $11.0 / 0.435$ & 3 \\
\hline $\mathrm{f} 622$ & $\uparrow$ & sen & $4.0 / 0.157$ & 5 \\
\hline $\mathrm{f} 628$ & $\rightarrow$ & $\begin{array}{c}\text { trian- } \\
\text { gular }\end{array}$ & $10.0 / 0.394$ & 5 \\
\hline $\mathrm{f} 630$ & $\rightarrow$ & sen & $2.3 / 0.092$ & 8 \\
\hline $\mathrm{f} 631$ & $\rightarrow$ & sen & $2.0 / 0.080$ & 10 \\
\hline $\mathrm{f} 632$ & $\rightarrow$ & $\begin{array}{c}\text { trian- } \\
\text { gular }\end{array}$ & $2.0 / 0.080$ & 4 \\
\hline $\mathrm{f} 633$ & $\uparrow$ & $\begin{array}{c}\text { trian- } \\
\text { gular }\end{array}$ & $2.0 / 0.080$ & 4 \\
\hline $\mathrm{f} 637$ & $\uparrow$ & $\begin{array}{c}\text { trian- } \\
\text { gular }\end{array}$ & $2.2 / 0.085$ & 8 \\
\hline $\mathrm{f} 640$ & $\rightarrow$ & $\begin{array}{c}\text { trian- } \\
\text { gular }\end{array}$ & $2.0 / 0.080$ & 8 \\
\hline
\end{tabular}

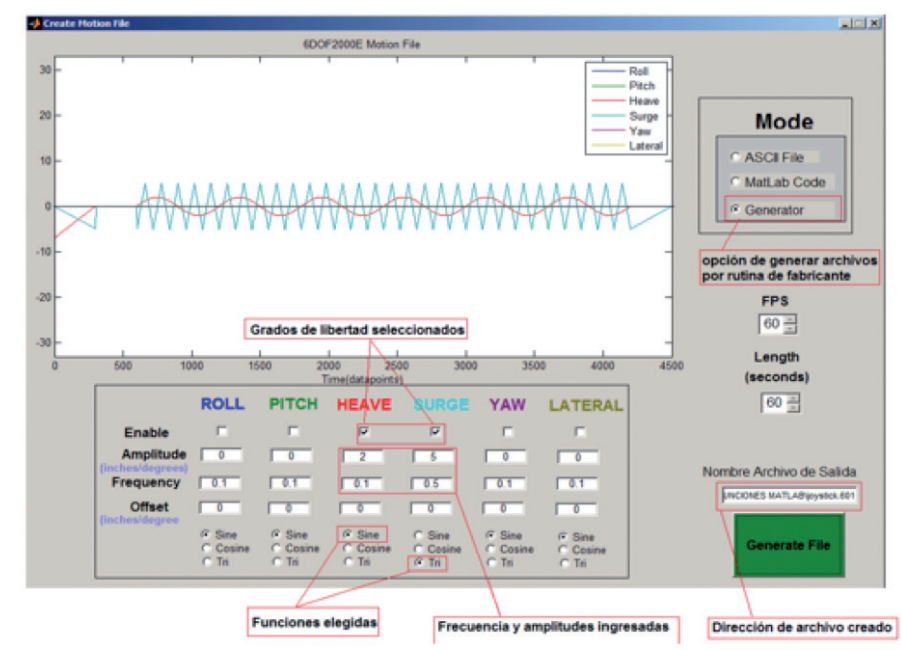

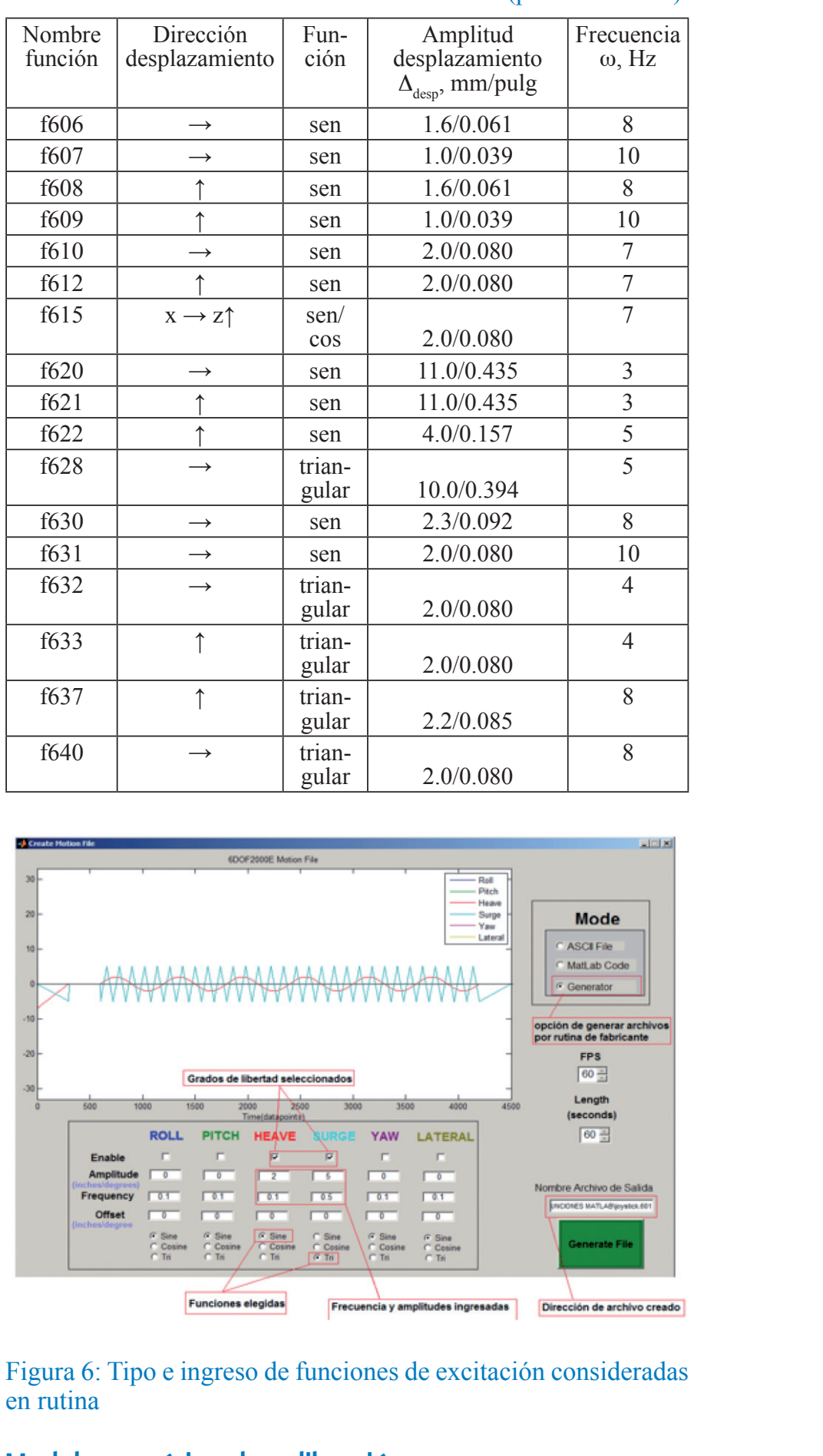

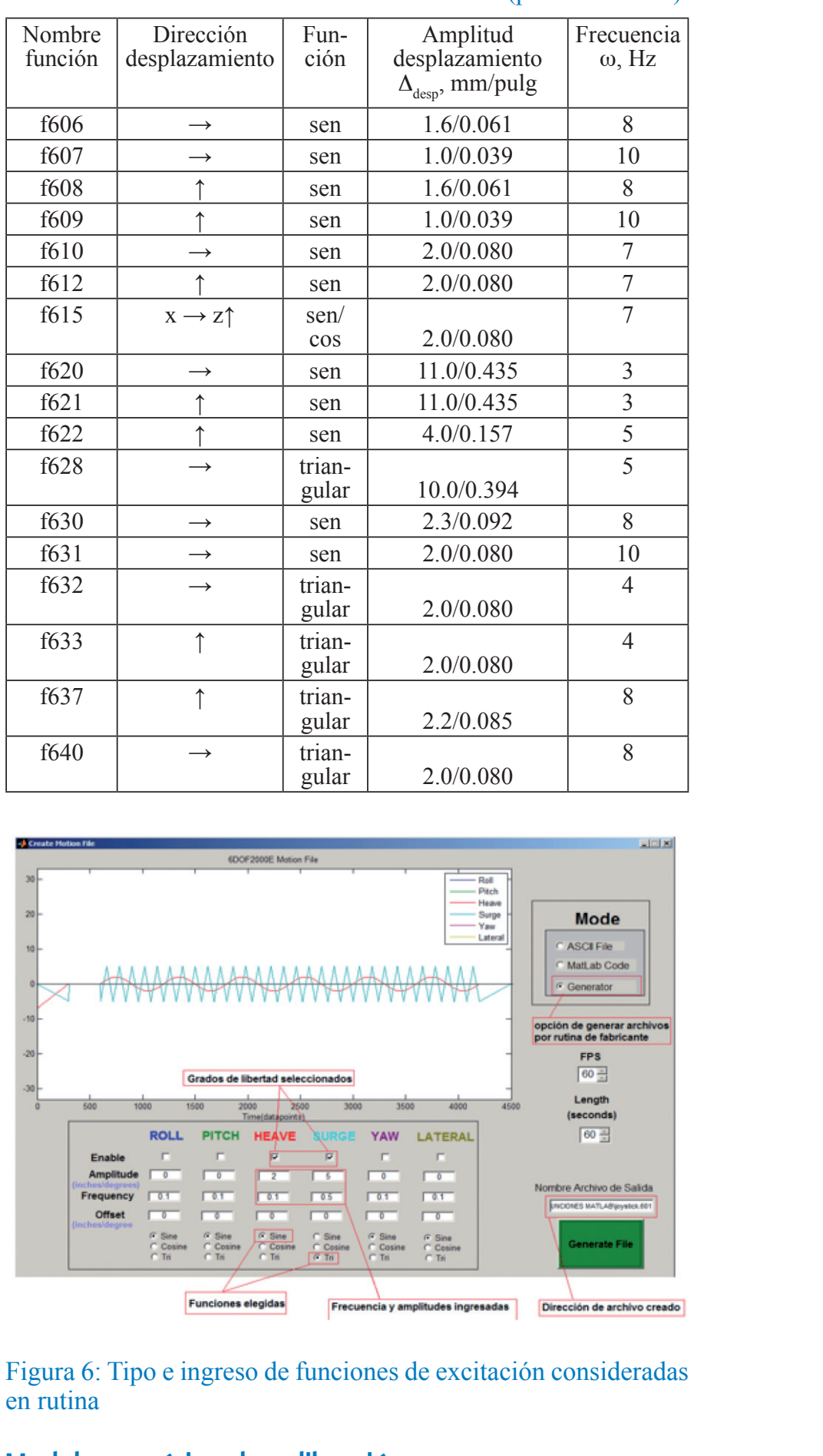

\section{Modelo numérico de calibración}

El modelo numérico es generado en base a antecedentes obtenidos de los ensayos de suelos realizados a las muestras utilizadas, complementados con datos obtenidos de la literatura en función de la clasificación del suelo resultante, como se indica en la Figura 8 y Tabla 2. Los valores de la Tabla 2 buscan ser representativos del suelo de fundación, arena de relave y lamas y corresponden a 
a)

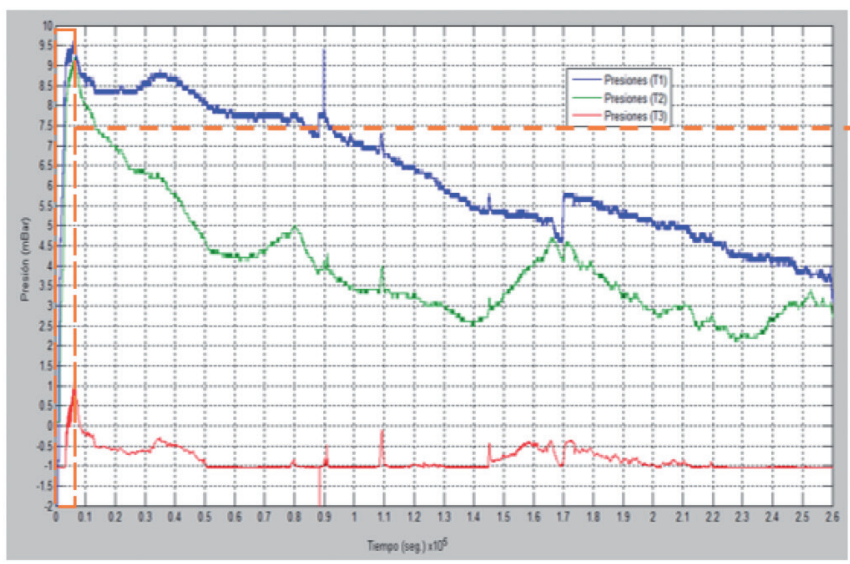

b)

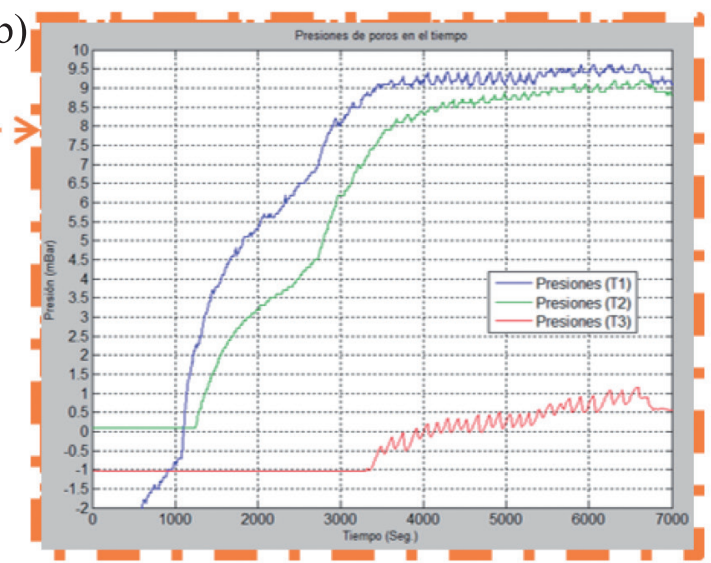

Figura 7: a) Presiones de poros para un periodo de 3 días, sin excitaciones en la base y b) acercamiento para los primeros $7000 \mathrm{~s}$

los adoptados en el modelo físico ensayado. Inicialmente los suelos en estudio no están saturados, esto es, donde se mide la presión de poros. Por lo tanto, existen condiciones parcialmente saturadas al comienzo, las cuales tienden a la saturación con el tiempo. Este fenómeno se denomina avance de un frente de saturación y su modelación numérica es más compleja que la abordada en este trabajo (e.g. Sinaba et al., 2013). El análisis del efecto de la matriz de succión de los suelos es un tema de investigación a realizar a futuro.

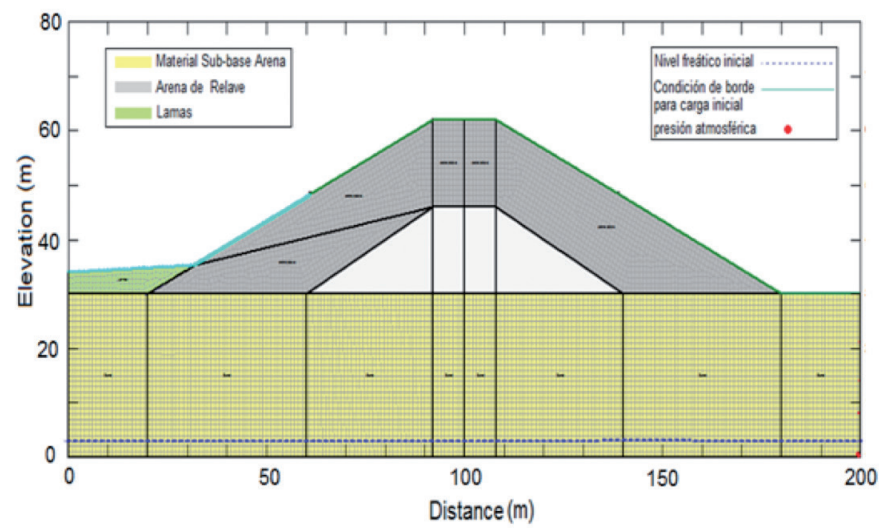

Figura 8: Esquema del modelo numérico

Tabla 2: Datos de entrada de los suelos, conductividad hidráulica saturada $k$, porosidad total $n$ y contenido de humedad residual $w_{r}$.

\begin{tabular}{|l|c|c|c|}
\hline Suelo & $k, \mathrm{~m} / \mathrm{s}$ & $n, \%$ & $w_{\mathrm{r}} \%$ \\
\hline $\begin{array}{l}\text { Arena de fundación SP con } \\
\text { poco fino }\end{array}$ & $3.5 \cdot 10^{-5}$ & 35 & 12 \\
\hline Arena de relave & $3.5 \cdot 10^{-6}$ & 35 & 25 \\
\hline Lamas & $2.0 \cdot 10^{-7}$ & 45 & 35 \\
\hline
\end{tabular}

\section{Resultados de simulación}

La simulación numérica se realizó para un periodo de 83 días, desarrollada en 2000 pasos de 3600 s cada uno, utilizando el programa SEEP/W (2012). El resultado del análisis entrega las presiones de poros para cada puerto de medición en función del tiempo. La Figura 9a muestra que las curvas de presiones de poros tienden a ascender con el tiempo en el puerto 1 y 2 , mientras que al pie del talud aguas abajo la presión de poros no aumenta con el tiempo. El ascenso las presiones de poros ocurre con tasas que van disminuyendo con el paso del tiempo, hasta tomar valores constantes, y desfasadas de acuerdo a cada tiempo que demora saturar cada puerto de medición.

Por otro lado, la Figura 9b muestra la variación con el tiempo del caudal en la interfaz agua embalsada y estrato de lamas; mostrando una curva decreciente, alcanzando un valor constante del orden de $1 \mathrm{~m}^{3} / \mathrm{h}$. La variación del caudal y posterior estabilización en $1 \mathrm{~m}^{3} / \mathrm{h}$ se debe a que, si bien es cierto la altura detrás de la presa es constante, la altura del agua delante de la presa varía y por ello hay una disminución del gradiente hidráulico que hace disminuir el caudal. Se aprecia que hay cierta similitud entre las presiones de poros obtenidas a través de la simulación numérica y las experimentales en cuanto al tipo de curva inicial (ver Figura 7b). No obstante, existe un efecto de escala temporal para la razón de cambio de las presiones de poros, el cual se calibra mediante un ajuste cinemático. Sin embargo, hay que destacar que, al analizar magnitudes y tiempo de las oscilaciones obtenidas experimentalmente, se debe escalar al tiempo real. 

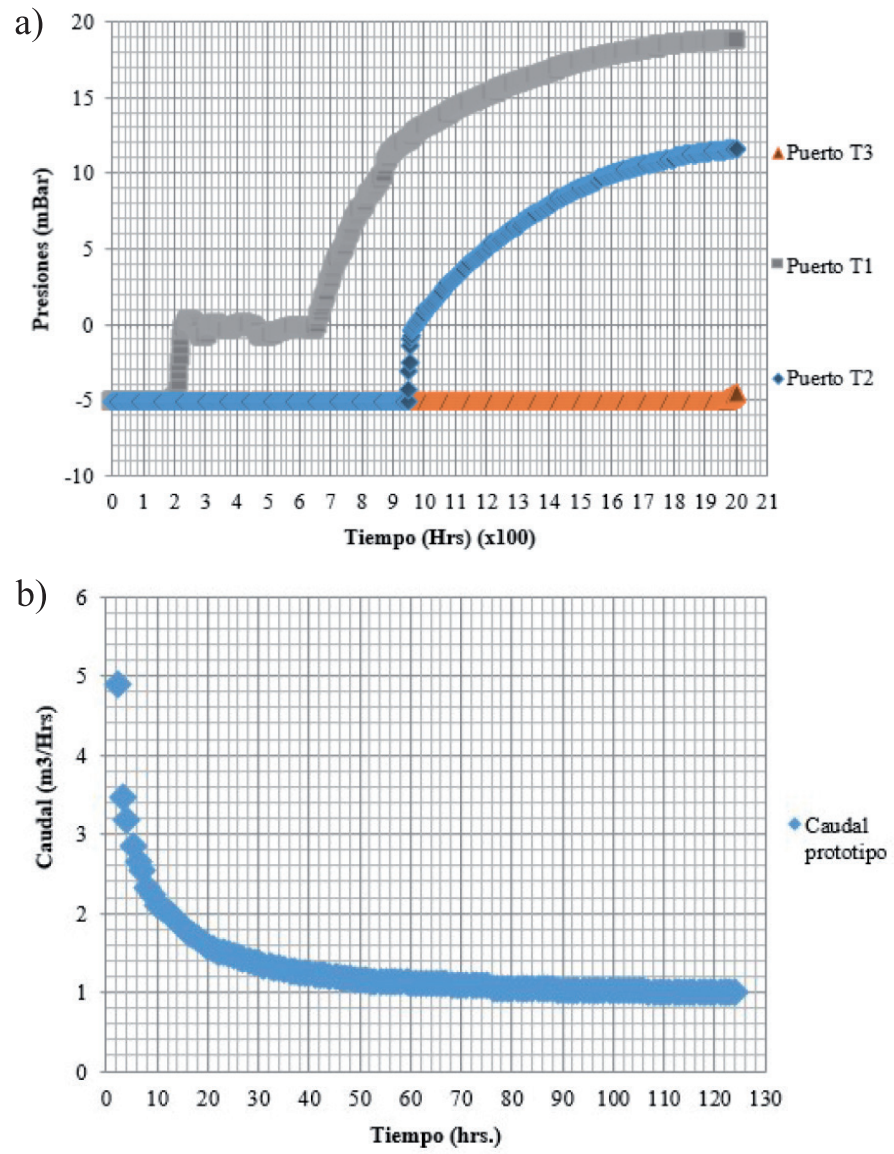

Figura 9: a) Respuesta de las presiones de poros y b) caudal infiltrante versus tiempo tras simulación

Se aprecia que hay cierta similitud entre las presiones de poros obtenidas a través de la simulación numérica y las experimentales en cuanto al tipo de curva inicial (ver Figura $7 b)$. No obstante, existe un efecto de escala temporal para la razón de cambio de las presiones de poros, el cual se calibra mediante un ajuste cinemático. Sin embargo, hay que destacar que, al analizar magnitudes y tiempo de las oscilaciones obtenidas experimentalmente, se debe escalar al tiempo real.

\section{Resultado de ensayo dinámico}

Se realizaron variados ensayos en base a las funciones presentadas en la Tabla 1, los cuales se hicieron en forma alternadas e incluso aleatoriamente en otros casos. Se presentan los casos de análisis más destacables del periodo 1 y 2 , donde se aprecian ciertas singularidades en el gradiente hidráulico y tendencias de las presiones de poros frente a determinados parámetros.

\section{Singularidades en la distribución de presiones}

\section{Periodo 1, mes de junio}

En la Figura 10a se observa que frente a las perturbaciones f609 y f608 se presenta un cambio significativo y progresivo en la variación de las presiones de poros. Se produce un ascenso de las presiones de poros en el puerto T3, llegando a superar las presiones del puerto T2 y alcanzando a las presiones del puerto T1 al final de la jornada. En la Figura $10 \mathrm{~b}$ se observa que inicialmente, previo a los ensayos, la presión de poros en T3 son las máximas de los 3 puertos y nuevamente $\mathrm{T} 2$ sigue siendo la mínima. La primera excitación f607, provoca un descenso casi brusco de las presiones, pero con retorno; luego frente a la perturbación f606, las presiones en T1 aumentan considerablemente y en forma progresiva. También se destaca dentro del mismo gráfico otro aumento de presiones de poros entre los 6000 y 8000 s, provocado por la función f607. Finalmente, en el gráfico de la Figura 10c, se aprecia una diferencia de

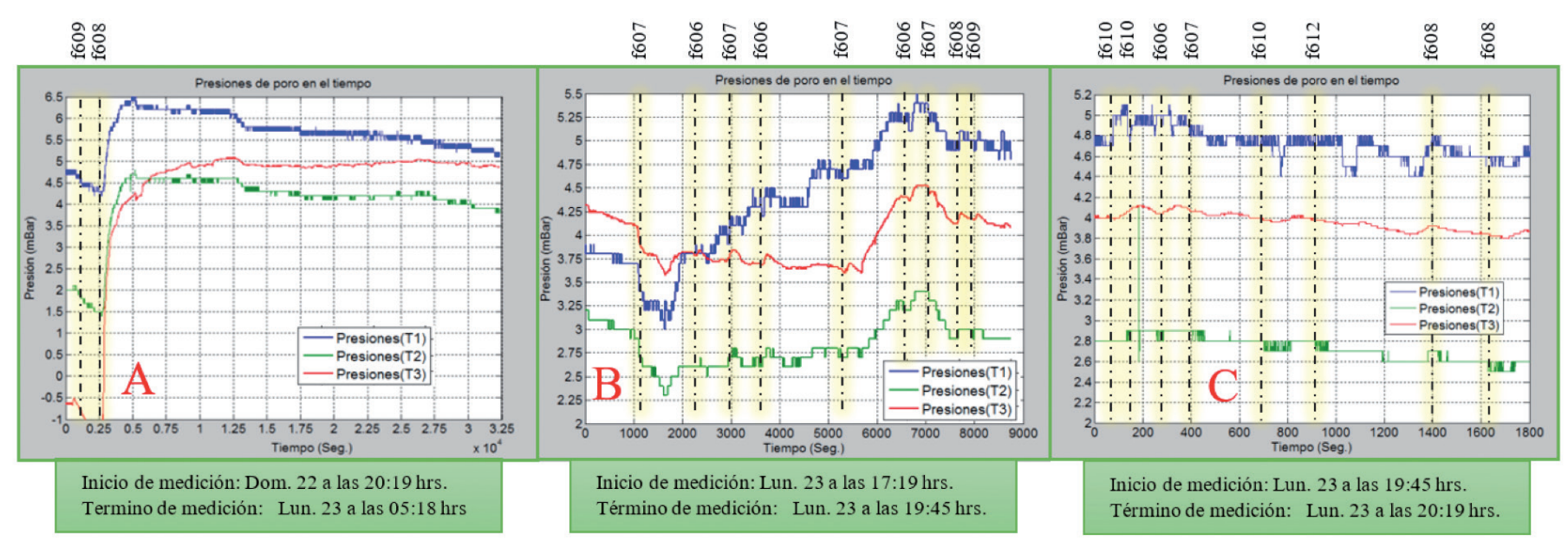

Figura 10: Comportamiento espacial-temporal de las presiones de poro para el mes de junio 


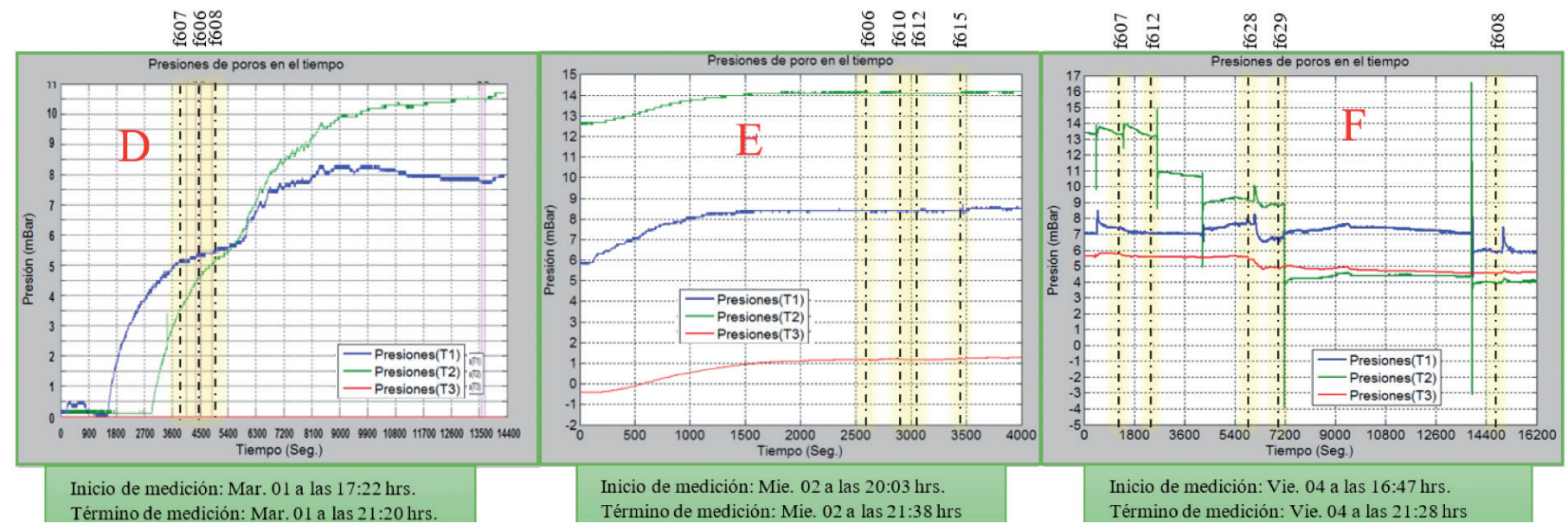

Figura 11: Comportamiento espacial temporal de las presiones de poro para el mes de julio

presiones de poros casi constante entre los 3 puertos y las presiones en el puerto 2, aún no han superado a las del puerto 3. Se destacan lagunas de oscilaciones instantáneas con retorno, especialmente en las presiones del puerto T1, mas no se observan otros cambios mayores.

\section{Periodo 2, mes de julio}

A diferencia del mes anterior, este periodo presenta un incremento de presiones en el puerto central T2. Analizando el gráfico de la Figura 11d; tras ejecutar las excitaciones f607, f606 y f608, se observa un ascenso de las presiones de poros en $\mathrm{T} 2$, superando a las del puerto $\mathrm{T} 1$ a los $5900 \mathrm{~s}$. Siguiendo cronológicamente con el gráfico de la Figura 11e, se aprecia que las presiones en T2 superan en magnitud a T1 y T2. Además, a partir de los $2000 \mathrm{~s}$ no hay variación temporal de las presiones de poros y se establecen los máximos valores para cada puerto T1, T2 y T3; esto es $8.5,14$ y 1.1 mbar, respectivamente. Las perturbaciones en este caso no muestran oscilaciones relevantes. El gráfico de la Figura 11f presenta oscilaciones instantáneas, específicamente caídas de presión de poros en el puerto T2. Otro fenómeno importante a destacar en el puerto T2, es que, a los $7200 \mathrm{~s}$, pasa de tener las mayores presiones de poros en el sistema, a tener nuevamente las mínimas como en el mes de junio.

\section{Variables influyentes en las magnitudes de oscilaciones piezométricas}

Las variaciones de las presiones de poros se pueden clasificar como variaciones tipo A como se muestra esquemáticamente en la Figura 12a. Esta variación corresponde a las respuestas de oscilaciones piezométricas que ocurren en forma instantánea a los ensayos tipo pulsaciones, las cuales solo manifiestan incrementos o decrementos locales sin alterar más allá el curso de las presiones en el tiempo. Las variaciones tipo B mostrada en la Figura $12 \mathrm{~b}$ representan a oscilaciones progresivas en el tiempo, las cuales a diferencia del anterior no se manifiestan como pulsos o peaks. Finalmente, variaciones tipo C mostradas en la Figura 12c, se refieren a cambios bruscos y permanentes en las presiones en el tiempo dentro del periodo de ensayo.
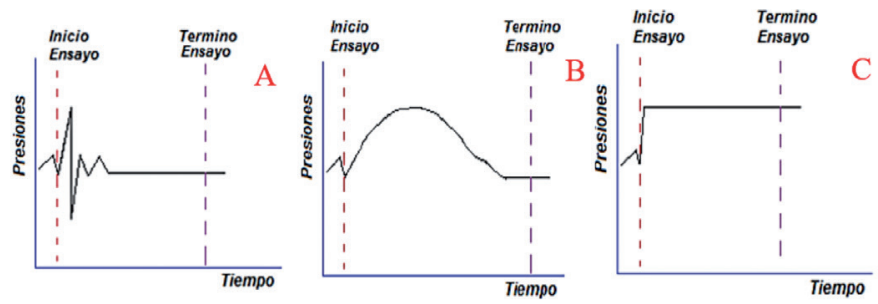

Figura 12: Esquema de variaciones de presiones, a) tipo A, b) tipo B y c) tipo C

Las variables influyentes que se eligen para el análisis de las oscilaciones piezométricas y que aparecen en la Tabla 2, son las siguientes:
a) Grado de saturación de todo el medio
b) Amplitud de desplazamiento
c) Posición del transductor (puertos T1, T2 y T3)
d) Tiempo transcurrido de análisis
e) Tipo de oscilación (A, B o C) 
Tabla 2: Variación de presiones de poros según amplitud de desplazamiento, tiempo transcurrido, posición de puerto, tipo de oscilación y grado de saturación inicial para el mes de julio

\begin{tabular}{|c|c|c|c|c|c|c|c|c|c|c|c|}
\hline \multirow{3}{*}{$\begin{array}{c}\text { Amplitud } \\
\text { (pulg.) }\end{array}$} & \multicolumn{10}{|c|}{ Varración de presiones (mBar) } & \multirow{3}{*}{ Grado deSaturación (\%) } \\
\hline & \multirow[b]{2}{*}{ Tempo } & \multicolumn{3}{|c|}{ Varracion Tipo A } & \multicolumn{3}{|c|}{ Varracion Tipo $B$} & \multicolumn{3}{|c|}{ Varracion Tipoc } & \\
\hline & & Puerto T1 & Puerto T2 & Puertot3 & Puertot1 & Puerto T2 & Puerto T3 & Puerto $\mathrm{T} 1$ & Puerto $T 2$ & PuertoT3 & \\
\hline \multirow{10}{*}{0,039} & 0,7 & 0,1 & 0,1 & 0 & 0 & 0 & 0 & 0,1 & 0,1 & 0 & 37,08 \\
\hline & 0,7 & 0,1 & 0,1 & 0 & 0 & 0 & 0 & 0,1 & 0,1 & 0 & 37,21 \\
\hline & 21,9 & 0,1 & 0 & 0 & 0 & 0 & 0 & 0,1 & 0 & 0 & 65,13 \\
\hline & 22,0 & 0,1 & 0 & 0 & 0 & 0 & 0 & 0 & 0 & 0 & 65,21 \\
\hline & 46,6 & 0,1 & 0,2 & 0,05 & 0 & 0 & 0,05 & 0 & 0,1 & 0 & 73,75 \\
\hline & 47,3 & 0,1 & 0,1 & 0 & 0 & 0 & 0 & 0,1 & 0,1 & 0,1 & 73,71 \\
\hline & 69,3 & 0,1 & 0,5 & 0,05 & 0 & 0,75 & 0 & 0,4 & 0 & 0,2 & 66,87 \\
\hline & 69,5 & 0,1 & 0,1 & 0,1 & 0 & 0 & 0,1 & 0 & 0,25 & 0 & 66,81 \\
\hline & 95,0 & 0,1 & 0 & 0,05 & 0,1 & 0,1 & 0 & 0,1 & 0 & 0 & 49,20 \\
\hline & 95.1 & 0.1 & 0.1 & 0 & 0.1 & 0.1 & 0 & 0 & 0 & 0 & 49.17 \\
\hline \multirow{10}{*}{0,0611} & 0.8 & 0,1 & 0,1 & 0 & 0 & 0 & 0 & 0,1 & 0,1 & 0 & 37,27 \\
\hline & 0.9 & 0,1 & 0,1 & 0 & 0 & 0 & 0 & 0,1 & 0,1 & 0 & 37,49 \\
\hline & 1,4 & 0,1 & 0 & 0 & 0 & 0 & 0 & 0,1 & 0,1 & 0 & 38,36 \\
\hline & 1.4 & 0,1 & 0.1 & 0 & 0 & 0 & 0 & 0,1 & 0.1 & 0 & 38.43 \\
\hline & 21,9 & 0,1 & 0 & 0 & 0 & 0 & 0,2 & 0 & 0,1 & 0 & 65,17 \\
\hline & 24,3 & 0,1 & 0,1 & 0,02 & 0 & 0 & 0,1 & 0 & 0 & 0 & 66,92 \\
\hline & 45,7 & 0,1 & 0,1 & 0.05 & 0 & 0 & 0 & 0 & 0 & 0 & 73,75 \\
\hline & 69,6 & 0,1 & 0,1 & 0,05 & 0,2 & 0,1 & 0,1 & 0 & d.5 & 0 & 66,76 \\
\hline & 95,1 & 0,1 & 0,1 & 0 & 0,1 & 0,1 & 0,1 & 0 & 0,1 & 0 & 49,13 \\
\hline & 95,1 & 0,1 & 0,1 & 0,1 & 1 & 0,25 & 0,2 & 0 & 0 & 0 & 49,12 \\
\hline \multirow{3}{*}{0,0787} & 95,3 & 0,1 & 0,2 & 0 & 0 & 0,15 & 0 & 0 & 0,05 & 0,1 & 48,98 \\
\hline & 95,5 & 0,1 & 0,1 & 0 & 0 & 0 & 0,1 & 0,1 & 0 & 0 & 48,79 \\
\hline & 95,6 & 0,05 & 0,1 & 0,05 & 0,05 & 0,1 & 0 & 0,1 & 0 & 0,1 & 48,75 \\
\hline \multirow{5}{*}{0,08} & 24,9 & 0,1 & 0,1 & 0.02 & 0 & 0 & 0,05 & 0 & 0 & 0 & 67,38 \\
\hline & 25,0 & 0,1 & 0,1 & 0,02 & 0 & 0 & 0,05 & 0 & 0 & 0,05 & 67,42 \\
\hline & 25,8 & 0,3 & 0,1 & 0.05 & 0,2 & 0 & 0 & 0,1 & 0 & 0.05 & 67,99 \\
\hline & 46,8 & 0,1 & 0,1 & 0.05 & 0 & 0.1 & 0,1 & 0.1 & 0,1 & 0.1 & 73,74 \\
\hline & 69.6 & 0.5 & 4 & 0,1 & 0 & 0 & 0,1 & 0.5 & 2 & 0 & 66,71 \\
\hline 0,0917 & 95,2 & 0,1 & 0 & 0,1 & 0,1 & 0 & 0,05 & 0 & 0 & 0 & 49,05 \\
\hline \multirow{2}{*}{0,1565} & 47,2 & 0,1 & 0,1 & 0,05 & 0,1 & 0 & 0,05 & 0,3 & 0.2 & 0,25 & 73,72 \\
\hline & 47,4 & 0,1 & 0 & 0,02 & 0,1 & 0 & 0,1 & 0 & 0 & 0 & 73,71 \\
\hline \multirow{4}{*}{0,3937} & 94,5 & 0 & 0 & 0 & 0.8 & 1 & 0 & 1.5 & 1.2 & 0,5 & 49,54 \\
\hline & 94,7 & 0,1 & 0,1 & 0,1 & 0,4 & 0,3 & 0,15 & 0 & 0 & 0 & 49,50 \\
\hline & 94,7 & 0,1 & 0,1 & 0,1 & 0,2 & 0 & 0,1 & 0 & 0 & 0 & 49,45 \\
\hline & 94,9 & 0,2 & 12 & 0,1 & 0.5 & 0 & 0,1 & 0 & 5 & 0.5 & 49,31 \\
\hline \multirow{3}{*}{0,4348} & 46,9 & 0,1 & ]$_{0,4}$ & 0,02 & 0 & 0 & 0 & 0.8 & 1 & 2.5 & 73,74 \\
\hline & 47,1 & 0,1 & 0,1 & 0,05 & 0,1 & 0 & 0,1 & 0 & 0 & 0 & 73,73 \\
\hline & 47,1 & 0,1 & 0.1 & 0.02 & 0.2 & 0 & 0 & 0 & $d .5$ & 1 & 73,72 \\
\hline
\end{tabular}

De los 2 periodos de ensayos, se elige el periodo 2 para discusión debido a que presenta mayores números de funciones empleadas, implicando un mejor seguimiento a la tendencia de las variaciones de presión. En la Tabla 2 aparecen enmarcadas tres partes, de las cuales se puede destacar que:

1) Se puede observar una mayor manifestación de oscilaciones de presiones de poro (en rojo), a medida que va aumentando la amplitud, que equivale decir a medida que la frecuencia va bajando.

2) A bajo grado de saturación, no hay manifestación alguna de presiones de poros para ningún tipo de oscilación.
3) Hay predominancia de oscilaciones tipo $\mathrm{C}$, lo que equivale a decir que no tiene implicancia el hecho que las perturbaciones en la base sean cíclicas y armónicas. Por lo tanto, no habría que esperar variaciones cíclicas de las presiones de poros.

\section{Comentarios finales}

El caso de mayor discusión corresponde al incremento y descenso de presiones de poros tras los ensayos dinámicos. Este fenómeno se manifiesta esencialmente en el puerto central de medición de presiones $\mathrm{T} 2$ el cual presenta 2 situaciones diferentes para cada periodo de ensayo. Descenso de presiones en el puerto T2 para el periodo 1 y aumento de presiones en el puerto T2 para el periodo 2 . 
Se puede afirmar que un descenso de presiones de poros en la zona central del tranque (puerto T2) ocurre cuando se genera succión (presiones de poros muy bajas o incluso negativas) por la oscilación del muro del tranque sobre la interfaz suelo de fundación - estructura. Esta interfaz puede encontrarse saturada mediante una lámina de agua que conecta la estructura y el subsuelo por efecto de tensión superficial. De ser así, la lámina puede generar succión al momento de la excitación dinámica, debido a que en esa interfaz todo vacío está ocupado tanto por agua como por aire. Por otra parte, en el periodo 2 ocurre el caso contrario al periodo anterior, a causa de que, en esta ocasión, la presión en el puerto T2 aumenta, lo cual era mucho más esperable y fácil de deducir que el primer caso. Este caso se puede justificar con el hecho de que las excitaciones basales aceleran el tranque de igual modo que en el primer caso, pero esta vez se transmite una sobrecarga la cual comprime al subsuelo, generando un aumento de las presiones de poros. Esto ha sido previamente mencionado por Custodio y Llamas (2001), refiriéndose a oscilaciones rápidas no periódicas, las cuales aluden a sobrecargas externas en un terreno. A modo de ejemplo, Custodio y Llamas (2001) mencionan la medición de presiones de poros en una vía férrea, en donde el tiempo de duración del incremento de presiones, es el mismo que el tiempo de estacionamiento del tren. Finalmente, se puede concluir que existe una relación entre la saturación del medio poroso y la respuesta de oscilaciones piezométricas. También hay una tendencia a que mayores amplitudes, lo que equivale a decir a menores frecuencias, tienden a incrementar la variación de las presiones de poros. Dentro de lo observado en la experimentación, destacan que estas bajas frecuencias generaban mayores ondas en el agua debido a que el modelo se desplazaba más, lo cual podría inferirse sólo como aproximación, que estas ondas también podrían transmitir sobrepresiones directas en proporción a sus amplitudes.

\section{Referencias}

Campaña, J., Valenzuela, L. and Bard, E. (2010). Seismic response of the Convento Viejo earth dam during the February $27^{\text {th }} 2010$ Chile earthquake. Obras y Proyectos 8, 37-46

Custodio, E. y Llamas, M.R. (2001). Hidrología subterránea. Segunda edición, vol. I, ediciones Omega, Barcelona
Figueroa, A., Solans, D., Gonzalez, C. y Campaña, J. (2017). Comportamiento sísmico de presas de relaves construidas aguas abajo y línea central. Obras y Proyectos 21, 30-37

Kissin, I.G., Belikov, V.M. and Ishankuliev, G.A. (1996). Shortterm groundwater level variations in a seismic region as an indicator of the geodynamic regime. Tectonophysics 265(3-4), 313-326

Koizumi, N., Kano, Y., Kitagawa, Y., Sato, T., Takahashi, M., Nishimura, S. and Nishida, R. (1996). Groundwater anomalies associated with the 1995 Hyogo-ken Nanbu earthquake. Journal of Physics of the Earth 44(4), 373-380

Martínez, M. (2010). Influencias de los eventos sísmicos en las aguas subterráneas. Tierra y Tecnología 37, 3-10

Pastén, M. (2016). Estudio experimental de la infiltración en un tranque de relaves sometido a una excitación dinámica basal. Tesis de título de Ingeniero Civil, Universidad Católica del Norte, Antofagasta, Chile

Roeloffs, E.A. (1998). Persistent water level changes in a well near Parkfield, California, due to local and distant earthquakes. Journal of Geophysical Research: Solid Earth 103(B1), 869-889

Rojstaczer, S. and Wolf, S. (1991). Hydrologic changes associated with the earthquake in The San Lorenzo and Pescadero drainage basins. Open file report 91-567, US Geological Survey, Menlo Park, California, 51-64

SEEP/W (2012). Seepage modeling with SEEP/W. An engineering methodology. July 2012 Edition. GEO-SLOPE International Ltd. Calgary, Canada

Shibata, T. and Akita, F. (2001). Precursory changes in well water level prior to the March, 2000 eruption of Usu Volcano, Japan. Geophysical Research Letters 28(9), 1799-1802

Sibson, R.H., Moore, J.M.M. and Rankin, A.H. (1975). Seismic pumping - a hydrothermal fluid transport mechanism. Journal of the Geological Society 131(6), 653-659

Sinaba, B., Becker, B., Klauder, W., Salazar, I. and Schüttrumph, H. (2013). On the proceeding of a saturation front under ponded conditions. Obras y Proyectos 13, 31-39

Valenzuela, L. (2016). Design, construction, operation and the effect of fines content and permeability on the seismic performance of tailings sand dams in Chile. Obras y Proyectos 19, 6-22

Valenzuela, L., Campaña, J., Bard, E. and Figueroa, A. (2016). High sand tailings dams: main challenges. Obras y Proyectos 20, 17-29 